\title{
Classification of Retinal Ganglion Cells: A Statistical Approach
}

\author{
Stephen M. Carcieri,* Adam L. Jacobs,* and Sheila Nirenberg \\ Department of Neurobiology, University of California, Los Angeles, California 90095-1763
}

Submitted 10 February 2003; accepted in final form 21 May 2003

Carcieri, Stephen M., Adam L. Jacobs, and Sheila Nirenberg. Classification of retinal ganglion cells: a statistical approach. J Neurophysiol 90: 1704-1713, 2003; 10.1152/jn.00127.2003. Numerous studies have shown that retinal ganglion cells exhibit an array of responses to visual stimuli. This has led to the idea that these cells can be sorted into distinct physiological classes, such as linear versus nonlinear or ON versus OFF. Although many classification schemes are widely accepted, few studies have provided statistical support to favor one scheme over another. Here we test whether some of the most widely used classification schemes can be statistically verified, using the mouse retina as the model system. We used a cluster analysis approach and focused on 4 standard response parameters: 1) response latency, 2) response duration, 3) relative amplitude of the ON and OFF responses, and 4) degree of nonlinearity in the stimulus-to-response transformation. For each parameter, we plotted its distribution and tested quantitatively, using a bootstrap method, whether it divided into distinct clusters. Our analysis showed that mouse ganglion cells clustered into several groups based on response latency, duration, and relative amplitude of the ON and OFF responses, but did not cluster into more than one group based on degree of nonlinearity - the latter formed a single, large, continuous group. Thus while some well-known schemes for classifying ganglion cells could be statistically verified, others could not. Knowledge of which schemes can be confirmed is important for building models of how retinal output is processed and how retinal circuits are built. Finally, this cluster analysis approach is general and can be used to test other classification proposals as well, both physiological and anatomical.

\section{N T R O D U C T I O N}

It is well known that retinal ganglion cells, the output neurons of the retina, do not respond in a uniform way to visual stimuli (Barlow 1953; Enroth-Cugell and Robson 1966; Hartline 1938; Kuffler 1953). When the retina is presented with almost any given stimulus, an array of responses is observed. For example, when ganglion cells are presented with flashing spots in their receptive field centers, some cells respond only to the onset of the spot, others only to the offset, and still others to both (Barlow 1953; Kuffler 1953). Similarly, when cells are presented with contrast-reversing sine wave gratings, some cells show linearity in their stimulus-to-response transformations, whereas others show striking nonlinearity (EnrothCugell and Robson 1966; Hochstein and Shapley 1976).

These findings have led to the idea that retinal ganglion cells fall into distinct classes based on their response properties (for review, see Rockhill et al. 2002; Stone 1983; Wassle and Boycott 1991). However, these findings do not necessitate the existence of distinct classes - that is, the fact that a particular

\footnotetext{
*S. M. Carcieri and A. L. Jacobs contributed equally to this work.

Address for reprint requests and other correspondence: S. Nirenberg, Dept. of Neurobiology, UCLA, 10833 Le Conte Ave., Los Angeles, CA 90095-1763 (E-mail: sheilan@ucla.edu).
}

response property varies from cell to cell does not imply that the cells divide into groups with respect to that property. Instead, the cells may just lie on a continuum (Abbott and Chance 2002; Hochstein and Shapley 1976; Mechler and Ringach 2002; Rodieck 1998). For example, cells with linear and very nonlinear responses may be endpoints of a continuum that contains cells with varying degrees of nonlinearity in between.

The issue of how ganglion cells divide-that is, what response properties divide them, and where the divisions liehas been a subject of much discussion and debate (reviewed in Rockhill et al. 2002; Rodieck and Brening 1983; Rowe and Stone 1977; Wassle and Boycott, 1991), and new proposals for relevant properties are continually emerging (e.g., see DeVries and Baylor 1997; O'Brien et al. 2002; Pang et al. 2002). One way to determine whether a given property divides cells is to use cluster analysis (Hochstein and Shapley 1976; Rodieck and Brening 1983; Rowe and Stone 1977). Although this approach has been used for molecular phenotyping of ganglion cells (Marc and Jones 2002), it has not been used for physiological classification. Here we applied a straightforward bootstrap algorithm to test for clusters in standard physiological response parameters.

The parameters chosen were among the most widely used for sorting ganglion cells: 1) response latency, 2) response duration, 3) relative amplitude of the ON and OFF responses, and 4) degree of nonlinearity in the stimulus-to-response transformation. Our aim was to determine how mouse ganglion cells divide with respect to these parameters because the answers have direct bearing on models of how retinal circuits are constructed and how retinal output is processed.

\section{METHOD S}

\section{Recording and stimulating}

Recordings were made from the isolated mouse retina using an extracellular multielectrode array as described previously (Nirenberg et al. 2001). Two stimuli were used. The first was a spot stimulus, which was used to examine response latency, duration, and the relative amplitude of ON and OFF responses. The stimulus consisted of a flashing spot that switched from light to dark every $2 \mathrm{~s}$ (light spot for $2 \mathrm{~s}$ followed by dark spot for $2 \mathrm{~s})$. The mean contrast between the light and dark spots, measured as $\left(L_{\max }-L_{\min }\right) /\left(L_{\max }+L_{\min }\right)$ where $L_{\max }$ is the maximum luminance and $L_{\min }$ is the minimum luminance, was 0.27 . The area of the screen not containing the spot was maintained at a background intensity equal to the mean of the light and dark spots

\footnotetext{
The costs of publication of this article were defrayed in part by the payment of page charges. The article must therefore be hereby marked "advertisement" in accordance with 18 U.S.C. Section 1734 solely to indicate this fact.
} 
$\left[1,400\right.$ rod-equivalent photons $\mu \mathrm{m}^{-2} \mathrm{~s}^{-1}$, placing the stimulus in the cone-activating regime with rods $>90 \%$ saturated (Dodd 1998)]. Each cell received an array of spots that varied in diameter from $50 \mu \mathrm{m}$ (the smallest that consistently drove the cells to fire) to $860 \mu \mathrm{m}$, in steps of $90 \mu \mathrm{m}$, and the different spot sizes were randomly interleaved. The cells in a given multielectrode recording were probed one at a time, with cells not receiving the stimulus kept at the background intensity. The total recording time for the spot stimulus ranged from 30 to 60 min: because multiple cells were recorded from simultaneously, and the spots that stimulated them were randomly interleaved (at any given moment, only one spot was present on the screen), the entire duration of spot stimulation depended on the number of cells in the recording. The spot stimulus lasted approximately $7 \mathrm{~min}$ for each cell and was presented to $4-8$ cells at a time, giving $30-60 \mathrm{~min}$ of stimulus.

The second stimulus was a series of contrast-reversing sine wave gratings (luminance modulated sinusoidally), which were used to examine the degree of nonlinearity in the stimulus-to-response transformation. The gratings were presented at 1 cycle/s ( 6 spatial frequencies, 12 phases, and 3 contrasts, with each combination presented for 10 cycles). The series was presented as follows: A spatial frequency and phase were chosen and presented at the 3 contrasts. The same frequency was then chosen again, but this time shifted in phase by $15^{\circ}$, and the 3 contrasts were shown again. This routine continued until the grating was shifted by a total of $180^{\circ}$; then a new spatial frequency was chosen. Spatial frequencies were presented in order from smallest to largest. The range ( 0.055 to 0.55 cycles/deg) was chosen, following Stone and Pinto (1993), to ensure detection of nonlinear spatial subunits within the receptive field center. The contrasts were $0.19,0.24$, and 0.27 . In this range, response amplitude increases linearly with contrast for most of the cells. Cells without a linear contrast-response relationship in this range were excluded (13\% of cells). Mean grating intensity was 1,400 rod-equivalent photons $\mu \mathrm{m}^{-2} \mathrm{~s}^{-1}$, as above. The grating stimulus runs for approximately 40 min, a time over which responses are typically extremely stable; stability is usually maintained for $>4 \mathrm{~h}$. To assess rundown, full-field flashes were presented before and after the grating stimulus. No statistically significant reduction in peak flash responses was observed $[P>0.5$, paired $t$-test comparing peak firing rates before and after gratings, $n=5$ retinas; average peak firing rate before gratings: $63 \pm$ 4 spikes/s (mean $\pm \mathrm{SE}$ ), after gratings: $66 \pm 4$ spikes/s].

\section{Finding the receptive field center of each cell}

For each cell, the receptive field was found using standard reverse correlation techniques (Meister et al. 1994). We presented a randomly flickering checkerboard stimulus with squares $70 \mu \mathrm{m}$ across that were either black or white, and whose intensity changed at random every 60 ms. Each cell's spike train was then reverse correlated to the stimulus, yielding an average movie that caused the cell to fire. This movie was denoted $I(\mathbf{x}, t)$, where $\mathbf{x}=(x, y)$ labels spatial position and $t$ labels time before a spike. The intensity was normalized so that $I=-1$ corresponded to a dark square and $I=+1$ corresponded to a light square. The maximum absolute value of the movie, denoted $I\left(\mathbf{x}_{\max }\right.$, $\left.t_{\max }\right)$, defines $t_{\max }$, the time at which the movie peaks. The center of the receptive field $\mathbf{x}_{\text {center }}$ was then set to the center of mass at the peak time

$$
\mathbf{x}_{\text {center }}=\frac{\sum_{\mathbf{x}} \mathbf{x} I\left(\mathbf{x}, t_{\max }\right)}{\sum_{\mathbf{x}} I\left(\mathbf{x}, t_{\max }\right)}
$$

where the sum on $\mathbf{x}$ is over only those intensities larger than threshold; that is, the sum contains intensities only for which $\left|I\left(\mathbf{x}, t_{\max }\right)\right|>$ $I_{\text {threshold }}$. The threshold intensity $I_{\text {threshold }}$ was taken to be 2.5 SDs above the noise, where the SD, $\sigma$, is defined by $\sigma^{2}=(1 / N) \Sigma_{x, t}$ $I(\mathbf{x}, t)^{2}$. Here, the sum is over all checkerboard squares and all time bins and $N$ is the total number of squares $\times$ the number of time bins.

\section{Finding the optimal spot for each cell}

For each cell, spots of various diameters were positioned over the cell's receptive field center, $\mathbf{x}_{\text {center }}$ (see Finding the receptive field for each cell) and then flashed on and off as described above. A spike density function for each spot diameter was computed by convolving the spike trains with a Gaussian of width $50 \mathrm{~ms}$. The peak of the spike density function was taken to be the cell's response. Response versus spot diameter for a typical cell is plotted in Fig. 1A. To find the peak of the response versus diameter curve, we fit a quadratic through three points: the spot with the largest response and spots one size smaller and one size larger. The peak of the quadratic provided an estimate of

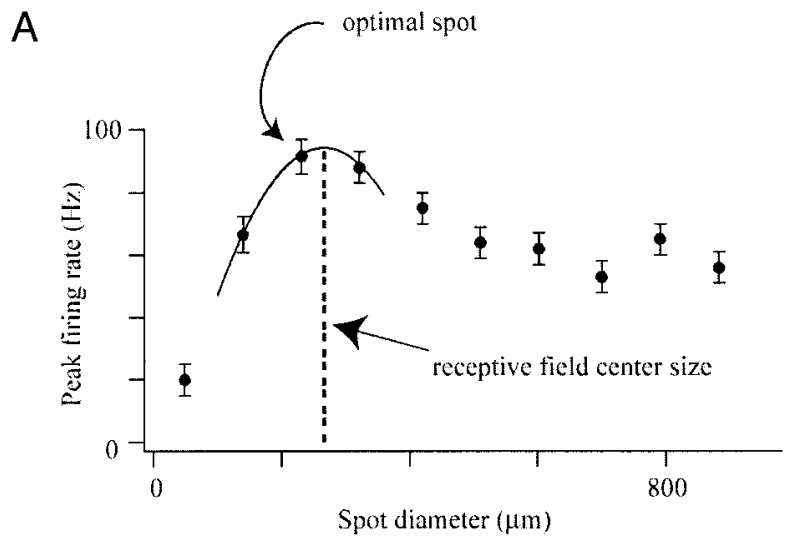

B

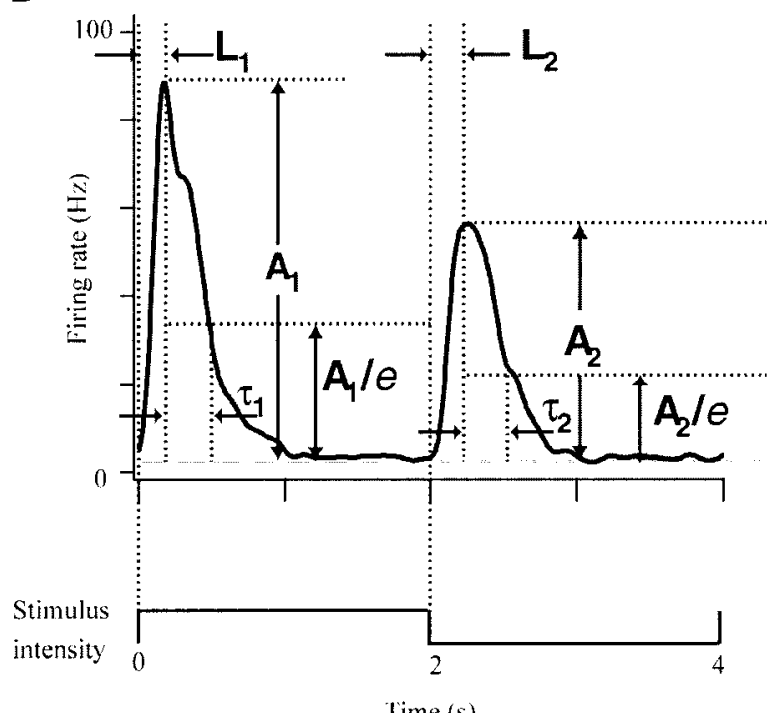

FIG. 1. Finding optimal spot and measuring parameters of response time course: amplitude, latency, and duration. A: series of spots of different sizes presented to ganglion cell receptive field center; peak response to each spot plotted as function of spot size. To find size of receptive field center, this function was fitted to a quadratic (solid line), using spot with largest response and spots on either side. Location of peak of quadratic (dotted line) provided estimate of receptive field center size. Largest spot smaller than this estimate was taken to be optimal spot. $B$ : measuring parameters of response time course, using optimal spot. Amplitude, $A_{i}$, is difference between peak response and baseline ( $i=1$ for response to light spot; $i=2$ for response to dark spot). Baseline firing rate (gray line) is computed by averaging firing rate over last $250 \mathrm{~ms}$ of each 2-s interval of stimulus. Latency, $L_{i}$, is time between stimulus onset (dark-to-light transition for $i=1$, and light-to-dark transition for $i=2$ ) and peak response. Finally duration, $\tau_{i}$, is time over which response $i$ falls from $A_{i}$ to $A_{i} / e$ above baseline. If decay were exponential, $\tau_{i}$ would be decay time constant. 
the receptive field center size. The largest spot smaller than this estimate was taken to be the optimal spot.

\section{Measuring response latency, bias index, and duration to the optimal spot stimulus}

The optimal spot was presented periodically and a spike density function was computed. Response latency, relative amplitude of the ON and OFF responses, and duration were measured (Fig. 1B). Latency, $L_{i}$, was defined as the time from stimulus onset to the peak of the spike density function ( $i=1$ for the response to the light spot; $i=2$ for the response to the dark spot). Relative amplitude of the ON and ofF responses was defined as a bias index, where the bias index is $\left(A_{1}-\right.$ $\left.A_{2}\right) /\left(A_{1}+A_{2}\right)$, and $A_{1}$ and $A_{2}$ are the peak responses of the first and second interval, respectively, relative to baseline. Baseline, shown as a gray line, was the firing rate averaged over the last $250 \mathrm{~ms}$ of both intervals. Duration, $\tau_{i}$, was the time over which the response decays from $A_{i}$ to $A_{i} / e$ above the baseline.

\section{Measuring the degree of nonlinearity}

Linearity of the stimulus-to-response transformation was evaluated using contrast-reversing sine wave gratings following the method of Hochstein and Shapley (1976) (Fig. 2). At each spatial frequency and phase, a peristimulus time histogram (PSTH) of the response was constructed using 10-ms bins, and the discrete Fourier transform of the PSTH was taken. At each spatial frequency, the ratio of the mean amplitude of the second harmonic across phases to the maximum amplitude of the first harmonic across phases was computed. (The amplitude of the second harmonic is, for the most part, invariant across phase, whereas the amplitude of the first harmonic varies sinusoidally; see Fig. 2.) The maximum second to first harmonic ratio with respect to spatial frequency was used as the nonlinearity index (Hochstein and Shapley 1976). The amplitude of the $n$th harmonic, $a_{n}$ was determined in the standard way

$$
a_{n}=\left|\frac{1}{N_{c}} \sum_{l=1}^{N_{c}} \frac{1}{C_{l} T_{\text {total }}} \sum_{j} v_{l}\left(t_{j}\right) \exp \left(-i n \omega t_{j}\right)\right|
$$

where $N_{c}$ is the number of contrasts presented, $C_{l}$ is the $l$ th contrast, $T_{\text {total }}$ is the total number of bins in the averaged response, $\omega$ is the temporal frequency $\left(2 \pi\right.$ radians/s), $v_{l}\left(t_{j}\right)$ is the average firing rate of bin $j$ in response to the $l$ th contrast, and $t_{j}$ is the time of bin $j$.

\section{Statistical analysis—the bootstrap test}

To determine whether a distribution forms more than one mode, we used the bootstrap algorithm described in Silverman (1981) with one addition: a normalization factor to prevent the development of false modes at boundaries [see Silverman (1986); for general review, see Efron and Tibshirani (1993)].

The following outlines the method. Consider a set of $n$ measurements, $z_{1}, \ldots, z_{n}$ (for example, $z_{i}$ might be the nonlinearity index of cell $i$, and $n$, the number of cells). The first step is to take these measurements and construct a smooth distribution. To do this, we treat each measurement as a $\delta$-function and convolve the set of $\delta$-functions with a Gaussian. The resulting smoothed distribution, denoted $f(t ; \mathbf{z}, h)$, is given by

$$
f(t ; \mathbf{z}, h)=\frac{1}{n} \sum_{i=1}^{n} \frac{1}{\Omega\left(z_{i}\right) \sqrt{2 \pi h^{2}}} \exp \left[-\frac{\left(t-z_{i}\right)^{2}}{2 h^{2}}\right]
$$

where $h$ is the width of the Gaussian and $\Omega\left(z_{i}\right)$ is a normalization factor that is needed whenever $t$ is restricted to a limited range (e.g., in the case of bias index, $t$ would lie in the range $[-1,1])$. If $t$ is limited to the range $[a, b]$, then $\Omega\left(z_{i}\right)=\left(2 \pi h^{2}\right)^{-1 / 2} \int_{a}^{b} \mathrm{~d} t \exp [-(t-$ $\left.z_{i}\right)^{2} / 2 h^{2}$ ]. If $t$ is not limited, then $a=-\infty, b=+\infty$, and $\Omega\left(z_{i}\right)=1$.

The second step is to determine how much the distribution has to be smoothed to make it unimodal. The smoothness is determined by the width of the Gaussian; thus we are asking how large $h$ must be to make $f(t ; \mathbf{z}, h)$ unimodal. If the set of measurements we started with was drawn from a unimodal distribution, then little smoothing will be needed ( $h$ will be small). If, on the other hand, the set of measurements we started with was drawn from a multimodal distribution, then a lot of smoothing will be needed ( $h$ will be large).

The third step is to set up the null hypothesis and determine whether it can be rejected. The null hypothesis is that the distribution is unimodal. To determine whether it can be rejected, we do the following: We find the smallest value of $h$ that makes $f(t ; \mathbf{z}, h)$ unimodal and denote this $h_{1}$. We then draw a sample of $n$ numbers from $f\left(t ; \mathbf{z}, h_{1}\right)$ and call these numbers $\hat{z}_{i}, i=1, \ldots, n$. We then find the smallest
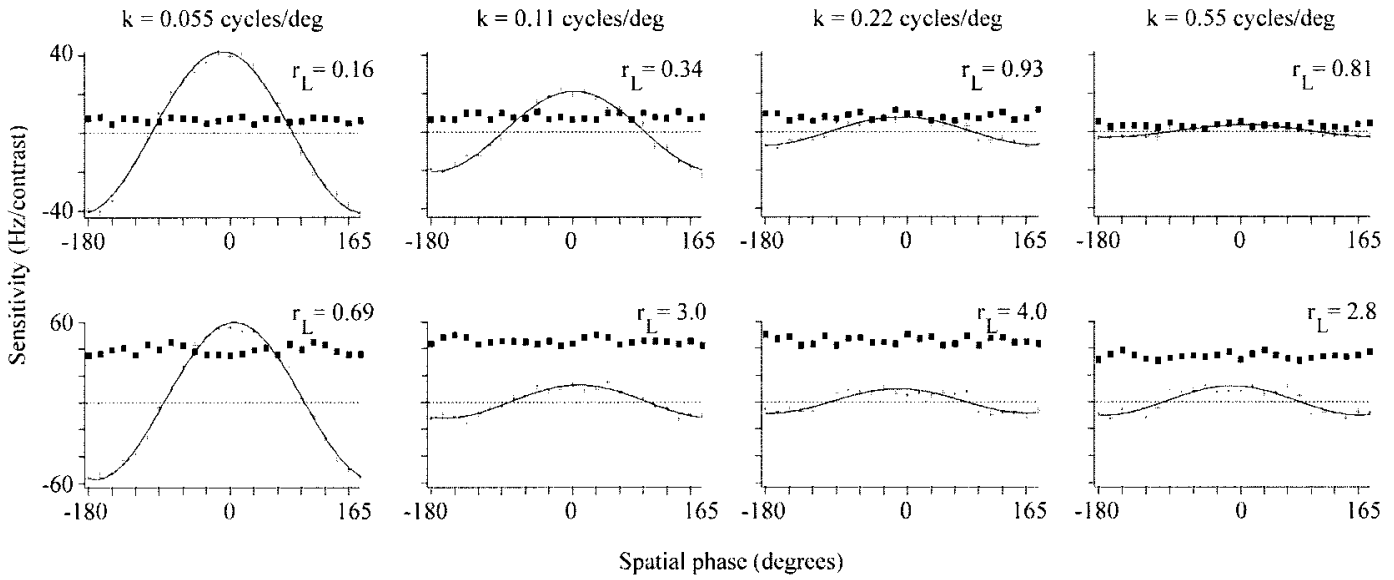

FIG. 2. Measuring degree of nonlinearity using Hochstein and Shapley's nonlinearity index (Hochstein and Shapley 1976). Amplitudes of first and second harmonic responses to contrast-reversing grating are shown as function of phase. Columns correspond to 4 different spatial frequencies; rows, to two different cells. First harmonic responses indicated by crosses; second harmonic responses, by squares. At low spatial frequencies first harmonic was a sinusoidal function of spatial phase, and sinusoids that fit those responses are shown (line). Sign of first harmonic response was chosen to ensure a continuous plot. Top cell exhibited a small second harmonic response, giving it a low nonlinearity index (index $=0.93$ ). Bottom cell exhibited a large second harmonic response, giving it a high nonlinearity index (index $=4.0$ ). Each plot is labeled with ratio of maximum first harmonic response to mean second harmonic response $r_{L}$. Maximum value of $r_{L}$ is nonlinearity index for cell. 
value of $h$ that makes $f\left(t ; \hat{\mathbf{z}}, h_{1}\right)$ unimodal. This value is denoted $\hat{h}_{1}$. We repeat this process $N$ times. The fraction of time $\hat{h}_{1}$ exceeds $h_{1}$ is the significance level for our test of the null hypothesis; in other words, the probability $P$ that our data came from a unimodal distribution is given by

$$
P=\left(\# \text { of times } \hat{h}_{1}>h_{1}\right) / N
$$

where $N$ is the number of bootstrap samples. If $P<0.05$, we reject the unimodal hypothesis and test the bimodal hypothesis using the same procedure. This process is repeated with successively higher modalities until we reach a number of modes that cannot be rejected. For example, to test for bimodality, we determine the smallest value of $h$ that makes $f(t ; \mathbf{z}, h)$ bimodal, denote this $h_{2}$, and then proceed as described above. For our analysis, we used $N=500$, which gave us an absolute error of $[P(1-P) / N]^{1 / 2}$ in our estimate of $P$. When $P=$ $0.05,[P(1-P) / N]^{1 / 2} \approx 0.01$.

A limitation of this method is that it assigns modes to single cells lying far from the main distribution. It is hard to know whether these single cells are outliers or reflect real modes, given that the sampling of the distribution is not dense enough. Rather than creating extra cell classes associated with the extra modes, we excluded isolated data points that were $>3$ SDs from the mean. On average, slightly fewer than $3 \%$ of the cells per retina were excluded as outliers.

\section{Serial approach to cell classification with respect to latency, bias index, and duration}

The most rigorous way to determine whether ganglion cells fall into distinct groups is to evaluate several parameters at once: that is, for $n$ parameters, construct an $n$-dimensional histogram and test for multimodality. Because the amount of data needed scales exponentially with $n$, a prohibitively large amount of data is needed even for 2 parameters. For this reason we evaluated parameters one by one in series. We first tested for multimodality with respect to one parameter, then moved onto the next, and so forth. If, at any stage, more than one mode appeared, then all modes were evaluated separately along the next parameter. The last stage was to backtrack-that is, to take the final set of modes and evaluate them for division on any previous parameters. For example, if the short latency group divided by duration, then each duration group was retested for division by latency. What finally stops the analysis is when a group cannot be further divided or it contains too few cells for further evaluation. (Our criterion, set arbitrarily, was that a group had to contain more than 20 cells.)

To explore as large a space as possible, we varied the order of parameters evaluated. This was done because some parameters were not measurable for all cells. For example, when we started with analysis of latency to the light spot, we found 3 cells that could not be evaluated because they showed no response to this spot. These cells were then lost for subsequent analysis because subsequent analysis focused on whether the modes found with respect to latency to the light spot further subdivided. To correct for this, we simply started the analysis with other parameters (e.g., latency to the dark spot). This way the cells eliminated by one order were included in another, and a different group was lost. We did this with all possible orders to find the largest number of modes for the data set. The order presented in RESULTS is just one of the many orders examined: it was chosen because 1 ) it revealed as many modes as were ever produced by any of the other orders and 2) no other order produced any type of mode it did not also produce.

All procedures on experimental animals were carried out under the regulation of the Animal Research Committee of the University of California at Los Angeles.

\section{RES ULTS}

\section{Sorting by response time course}

Each ganglion cell was presented with the standard stimulus for evaluating response time course, a flashing spot that targeted only the receptive field center (see METHODS). The stimulus was periodic and consisted of a light spot presented for $2 \mathrm{~s}$, followed by a dark spot presented for $2 \mathrm{~s}$. Three standard parameters associated with response time course were then measured: the latency of the response to the light and dark spots, the relative amplitude of the responses to the light and dark spots, and the duration of the response to the light and dark spots. Finally, the distribution of each parameter was tested for multimodality. Division into more than one mode would indicate that the cells divide naturally into more than one class.

Multimodality was examined using a bootstrap algorithm (Silverman 1981). With this method, the distribution is first tested for unimodality. If this is rejected, the distribution is then tested for bimodality. The process is repeated using successively higher modes, until the number of modes that cannot be rejected is identified (see METHODS).

The first parameter examined was the latency of the response to the light spot. Latency was defined as the time between spot onset and peak response (see METHODS, Fig. 1B). The distribution of latencies was found to be bimodal: unimodality was rejected $(P<0.01)$; bimodality was not $(P=0.25 ; n=108)$ (Fig. 3). This indicates that ganglion cells divide into two latency classes: one containing short latency responses $(<400$ $\mathrm{ms}$ ) and one containing long latency responses ( $>400 \mathrm{~ms})$.

These classes were then further evaluated for division by the latency of their responses to the dark spot. No such divisions were found: unimodality was not rejected for either class $(P=$ $0.26, n=43$ for the short latency class; $P=0.08, n=37$ for the long latency class) (Fig. 4).

Unimodality
rejected; $p<0.01$

Bimodality not rejected; $p=0.25$

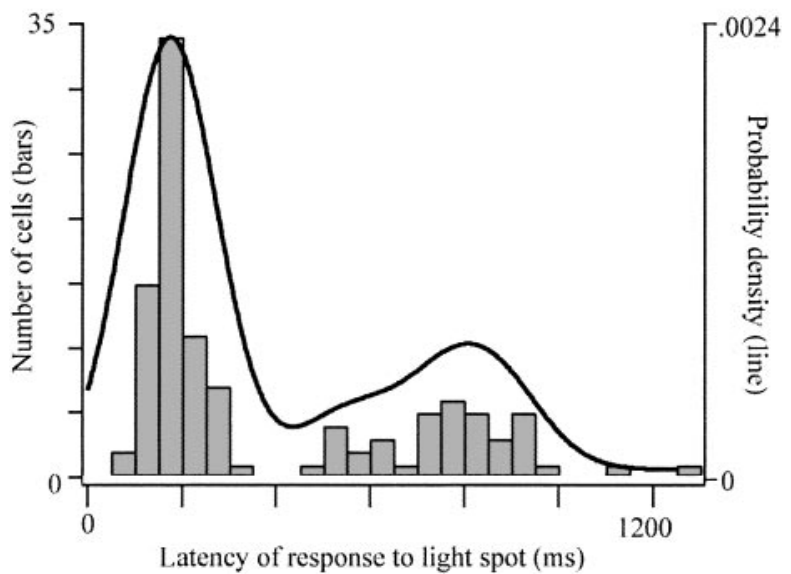

FIG. 3. Histogram of response latencies using light spot. Smoothed distribution is overlaid (line). Unimodality was rejected $(P<0.01)$; bimodality was not $(P=0.25 ; n=108)$, indicating two latency groups. Note that test for multimodality is independent of bin size; binned histogram is just for visualizing data. 
Next, the classes were examined for division attributed to differences in the relative amplitude of their responses to the light and dark spots. This was measured using a bias index, which is the amplitude of the response to the light spot minus the amplitude of the response to the dark spot divided by their sum (see METHODS, Fig. $1 B$ ). It is +1 for cells that respond only to the light spot and -1 for cells that respond only to the dark spot.

Our analysis showed that the short latency class did, in fact, subdivide by bias index: unimodality and bimodality were rejected $(P<0.02$ and $P<0.002$, respectively), but trimodality was not $(P=0.52, n=70)$ (Fig. $5 A)$. This indicates that the short latency class divides into 3 bias index classes: one containing cells with a bias index near +1 (on cells), one containing cells with a bias index near -1 (OFF cells), and one containing cells with a bias index near 0 (ON-OFF cells). The long latency class, on the other hand, did not subdivide: unimodality was not rejected $(P=0.15, n=38)$ (Fig. 5B).

The analysis of latency and bias index revealed 4 classes: short latency and long latency cells, with the short latency cells dividing into ON, OFF, and ON-OFF classes. These classes were then examined for further subdivision on the basis of differ-
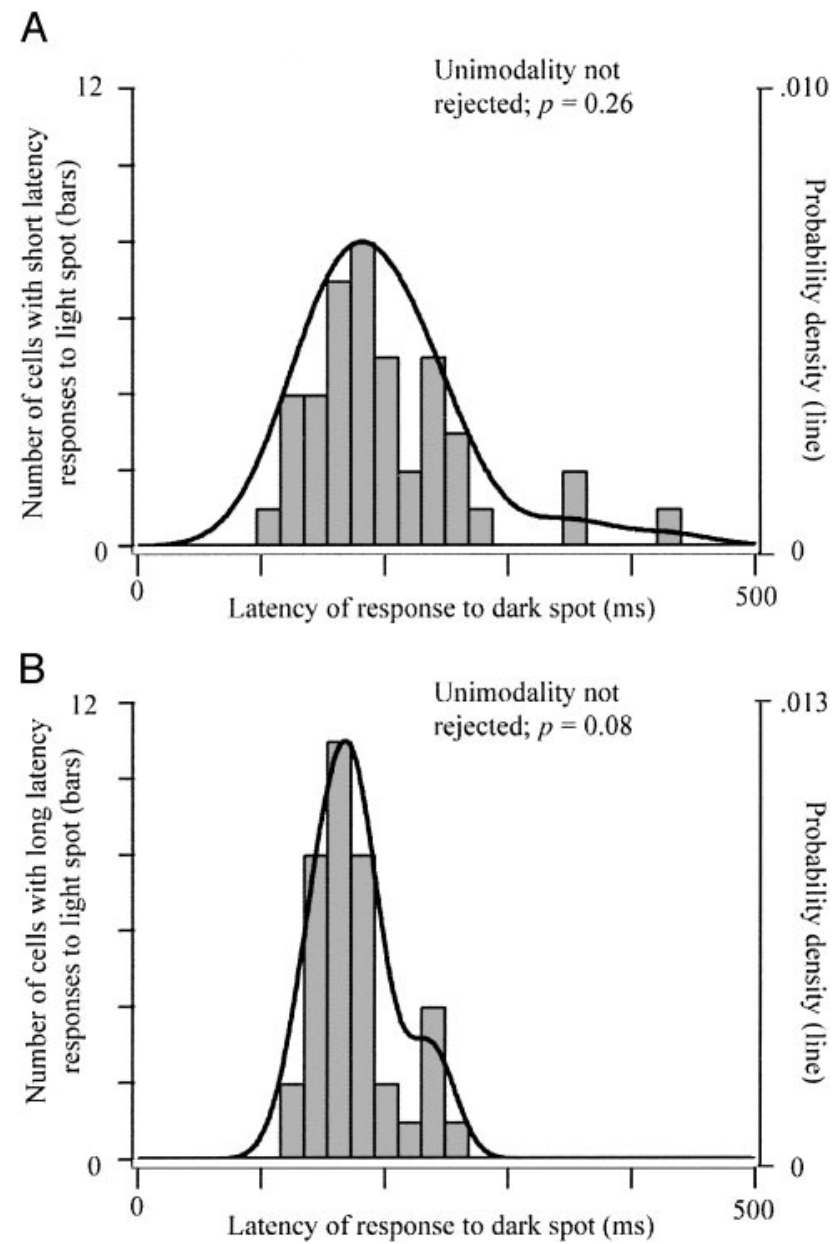

FIG. 4. Histograms of response latencies using dark spot. The two groups found in Fig. 3, that is, the group with short latency responses to light spot and the group with long latency responses to light spot, were evaluated separately. $A$ : histogram for cells with short latency responses to light spot. Unimodality was not rejected $(P=0.26 ; n=43) . B$ : histogram for cells with long latency responses to light spot. Unimodality was not rejected $(P=0.08 ; n=37)$.

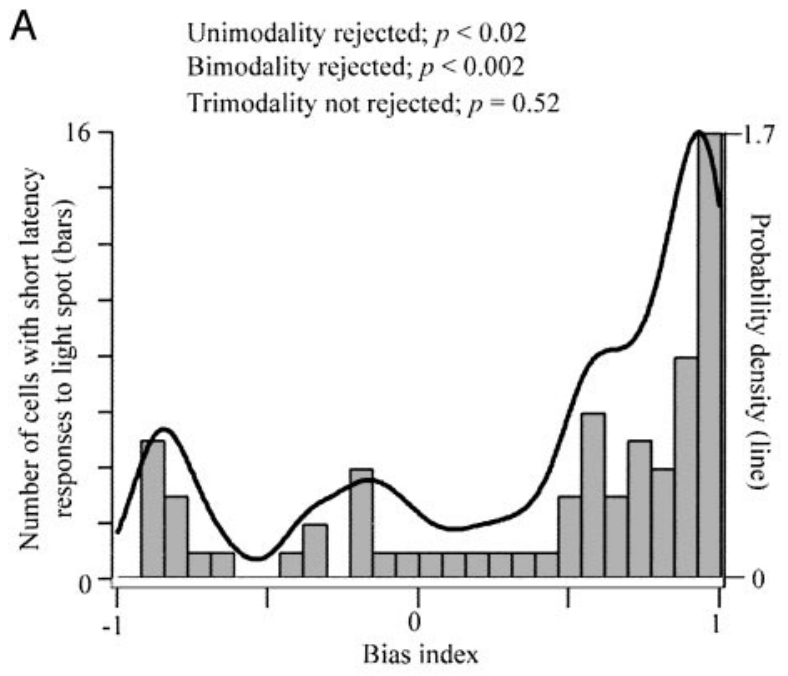

B Unimodality not rejected; $p=0.15$

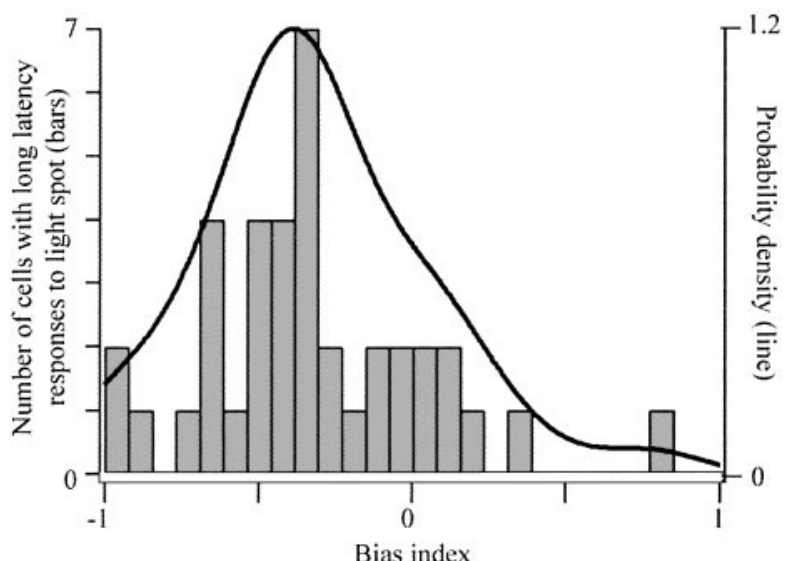

FIG. 5. Histograms of bias index, evaluated separately for groups found in Fig. 3. A: histogram for cells with short latency responses to light spot. Unimodality was rejected $(P<0.02)$; bimodality was rejected $(P<0.002)$; trimodality was not rejected $(P=0.52 ; n=70)$. This indicates that the distribution divides into 3 groups: OFF, ON-OFF, and ON groups. $B$ : histogram for cells with long latency responses to light spot. Unimodality was not rejected $(P=0.15, n=38)$. The distribution shows more ON than OFF cells or ON-OFF cells, consistent with previous observations, both in vivo (Balkema and Pinto 1982) and in vitro (Nirenberg and Meister 1997). Stone and Pinto (1993) also observed more ON than OFF cells, but to a lesser extent than in these other studies.

ences in response duration. Duration of the response to the light spot was examined first. This was measured as the time from peak response to $1 / e$ of the peak (see METHODs, Fig. $1 B$ ). Of the 4 classes, only 2 had a sufficient number of cells for this analysis ( 49 cells in the short latency on class and 38 cells in the long latency class. The short latency off class and the short latency ON-OFF class contained 10 and 11 cells, respectively). Our analysis showed that the short latency on class subdivided: unimodality was rejected $(P<0.02)$, bimodality was not $(P=$ $0.78, n=46$ ) (Fig. 6A). This indicates that the short latency ON class divides into 2 duration classes: one with responses $<200$ $\mathrm{ms}$ (transient cells) and one with responses $>200 \mathrm{~ms}$ (sustained cells). The long latency class did not subdivide: unimodality was not rejected $(P=0.24, n=38)$ (Fig. $6 B)$.

The short latency on class and the long latency class were then examined for division by response duration to the dark 

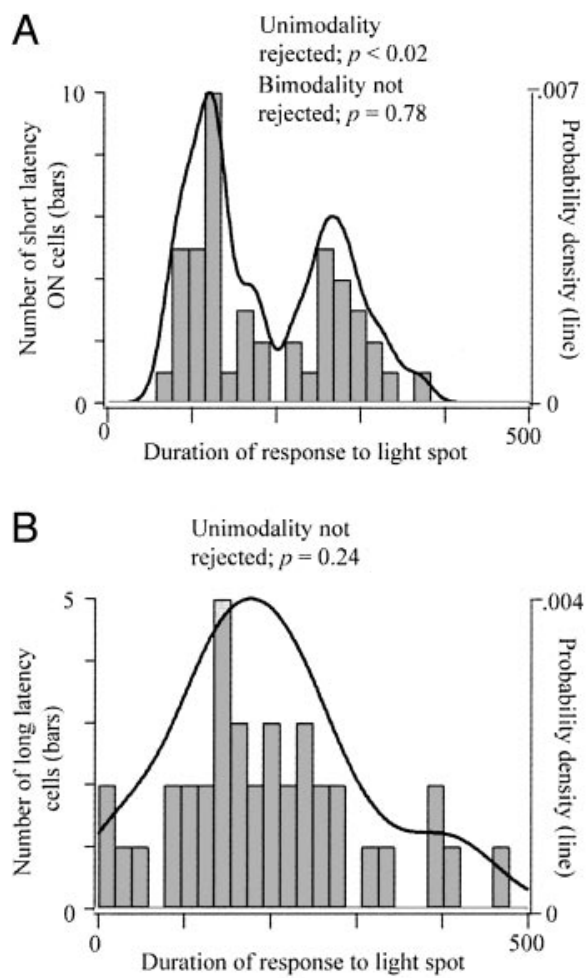
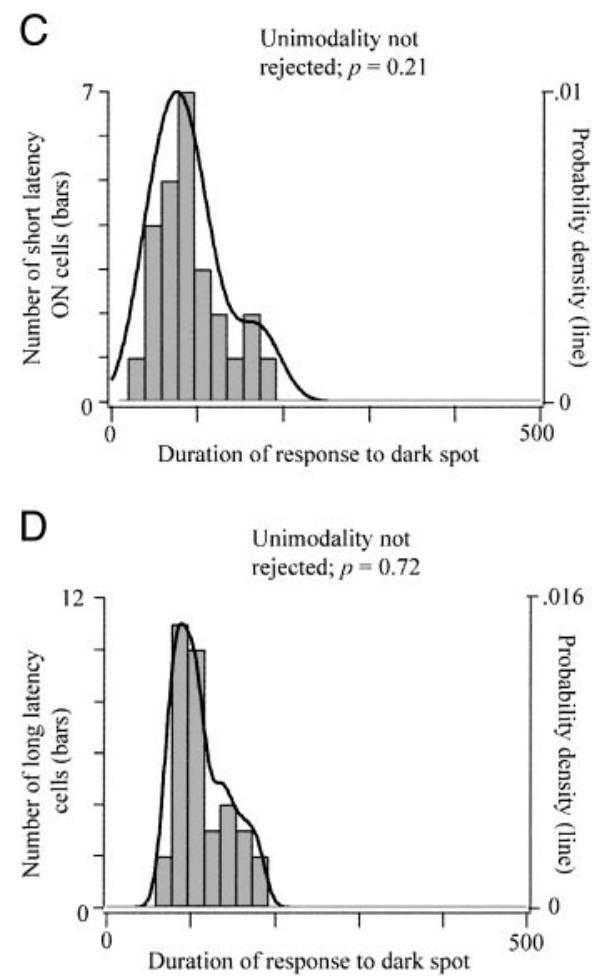

FIG. 6. Histograms of response durations using light and dark spots, evaluated separately for 2 of 4 groups found in Fig. 5: short latency ON cells and long latency cells. Other 2 groups had too few cells for evaluation (see RESULTS). A: histogram of response durations to light spot for short latency oN cells. Unimodality was rejected $(P<0.02)$; bimodality was not rejected $(P=0.78 ; n=46)$. This indicates that the distribution divides into 2 groups, one with transient responses and one with sustained responses. $B$ : histogram of response durations to light spot for long latency cells. Unimodality was not rejected $(P=0.24, n=38)$. $C$ : histogram of response durations to dark spot for short latency on cells. Unimodality was not rejected $(P=0.21, n=26)$. $D$ : histogram of response durations to dark spot for long latency cells. Unimodality was not rejected $(P=0.72, n=$ $35)$. spot. No divisions were found: unimodality was not rejected for either group $(P=0.21$ for the short latency on cells, $n=$ 26; $P=0.72$ for the long latency cells, $n=35$ ) (Fig. 6, $C$ and $D$ ).

Finally, the last stage of analysis was reached. The preceding step brought the total number of classes up to 5 , given that the short latency class divided into 2 duration groups. The last stage was to take the 5 classes and back track-that is, test whether they produce any further subdivision on the basis of differences in parameters previously tested. All classes were examined for all possible subdivisions with these parameters, and no new divisions emerged: unimodality was not rejected for any class $(P>0.25$ for all $)$.

Taken together, our analysis of latency, bias index, and response duration revealed 5 classes of ganglion cells. They are short latency, transient on cells; short latency, sustained on cells; short latency ON-OFF cells; short latency OFF cells; and long latency cells (Fig. 7). To be certain that no other classes were present (see DISCUSSION), we performed the analysis with different parameter orders (e.g., duration to the dark spot, then latency to the light spot, etc.; see METHODS.) All possible orders were evaluated and no additional groups emerged.

\section{Sorting by the degree of nonlinearity in the stimulus-to-response transformation}

To evaluate the degree of nonlinearity in the stimulus-to response transformation, the retina was presented with contrast-reversing sine wave gratings, the responses of the ganglion cells were Fourier analyzed, and the degree of nonlinearity was measured using the classic nonlinearity index developed by Hochstein and Shapley (1976). This index measures the strength of the second harmonic of the response relative to the first (see METHODS, Fig. 2). An index of zero indicates a stimulus-to-response transformation that is essentially linear, and any value above zero indicates some nonlinearity. Finally, the distribution of nonlinearity indices for all cells in our data set was plotted and evaluated for multimodality.

Our analysis showed that the distribution of nonlinearity indices was unimodal-that is, unimodality could not be rejected $(P=0.43, n=137)$. Although the distribution showed a broad range of indices, from near 0 to as high as 9 (Fig. 8A), there was no gap dividing the cells into distinct linear and

$$
\text { LATFNCY BIAS DURATION CLASS }
$$

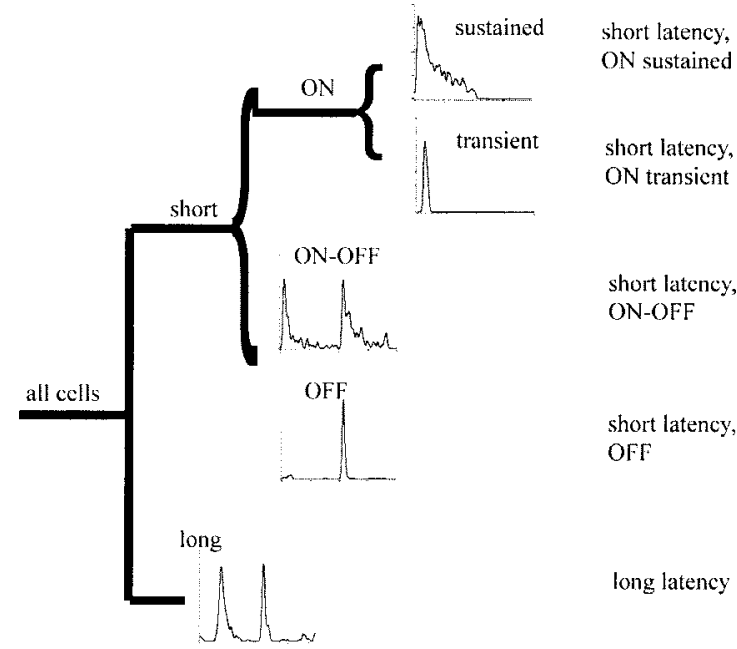

FIG. 7. Ganglion cells divide naturally into five classes using spot stimulus. Latency to light spot divides cells into two classes, long latency and short latency. Bias index then divides short latency class into ON, OFF, and ON-OFF classes. Duration of response to light spot then further subdivides short latency ON cells into transient and sustained classes. 
Unimodality not rejected; $\mathrm{p}=0.43$

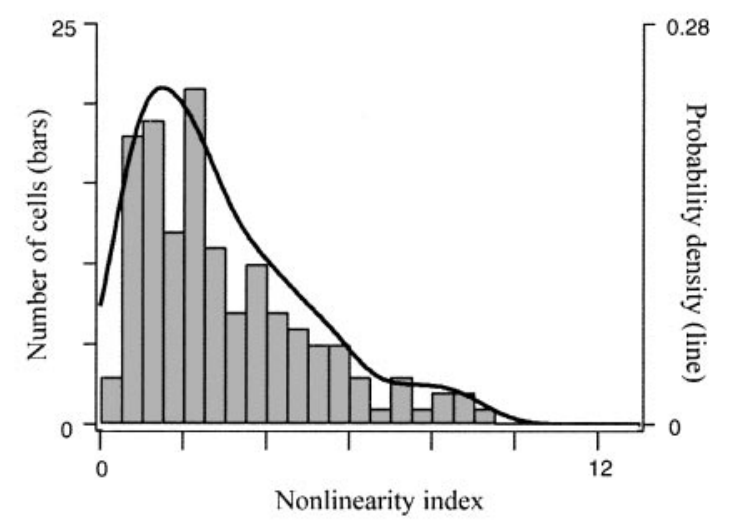

B Unimodality not rejected; $\mathrm{p}=0.07$

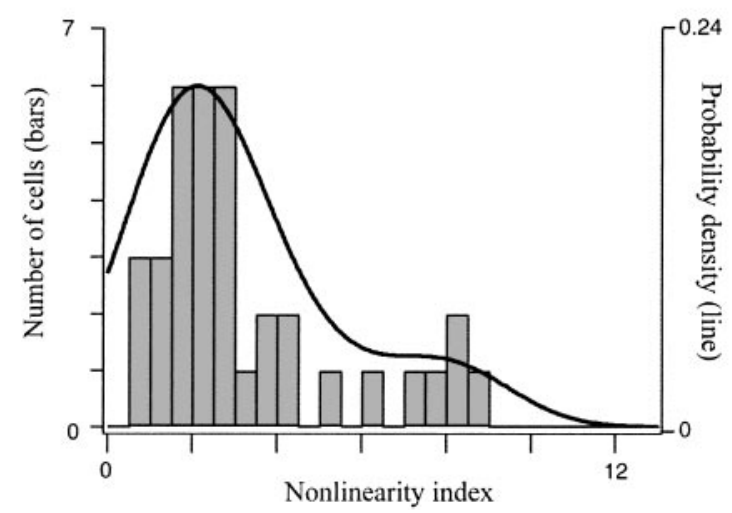

FIG. 8. A: histogram of nonlinearity indices. Smoothed distribution is overlaid (line). Unimodality was not rejected $(P=0.43 ; n=137)$, indicating there are no distinct linear and nonlinear groups. $B$ : histogram of nonlinearity indices for subset of ganglion cells all found at similar, central retinal eccentricity (within $600 \mu \mathrm{m}$ of optic nerve head). As with larger data set in $A$, unimodality was not rejected $(P=0.07 ; n=36)$, indicating that linear and nonlinear cells form a continuum even when evaluated at one eccentricity. Note that any potential gaps in the distribution occur at index values well above 1, the value that would be expected to separate linear from nonlinear cells.

nonlinear groups. Thus there was a continuum, with cells showing varying degrees of nonlinearity in their responses.

We addressed this further by testing whether the distributions at each spatial frequency showed multimodality and found, as above, that no significant divisions were present (unimodality could not be rejected in all cases: at 0.055 cycles/ deg, $P=0.07$; at 0.11 cycles $/ \mathrm{deg}, P=0.57$; at 0.165 cycles/ $\operatorname{deg}, P=0.31 ;$ at 0.22 cycles $/ \mathrm{deg}, P=0.32$; at 0.275 cycles/ deg, $P=0.18$; at 0.55 cycles $/ \mathrm{deg}, P=0.35$ ). Note that for this analysis "nonlinearity index" means simply the ratio of the mean of the second harmonic to the maximum of the first harmonic at a given spatial frequency. The nonlinearity index used in the preceding paragraph is the maximum of this ratio across all spatial frequencies.

\section{I S C U S S I O N}

The issue of how ganglion cells divide into distinct classes has long been a subject of debate. This issue has been difficult to resolve because the criteria used to define cell classes are often qualitative. Many classifications are defined primarily by descriptions of a typical example of each class, leaving atypical or borderline examples difficult to classify. In addition, when the criteria are qualitative, they are to some extent dependent on the interpretations of the investigator, creating the possibility that classifications may vary over time or between laboratories. Our aim was to build on the theoretical work of Hochstein and Shapley (1976), Rodieck and Brening (1983), and Rowe and Stone (1977), and lay out a straightforward method, extracted from the statistical literature (Silverman 1981), for testing which parameters divide cells into discrete groups.

Our approach was to take a physiological response parameter that had been previously used to group cells (e.g., the degree of nonlinearity in the stimulus-to-response transformation), plot the distribution of that parameter (e.g., the distribution of nonlinearity index values for all cells in the data set), and then test the distribution for multimodality. The null hypothesis in each case was that the distribution was unimodal. If unimodality was rejected, then the distribution was tested for bimodality, and so forth. With this approach, one can obtain definitive evidence, that is, statistically significant evidence, that the cells divide into distinct groups with respect to that parameter, and identify where the divisions lie.

We first tested whether ganglion cells divide into distinct on, OFF, and ON-OFF classes; whether they sort into transient and sustained types; and whether they form short and long latency groups, three widely used classification schemes (Balkema and Pinto 1982; Barlow 1953; Cleland et al. 1971; DeVries and Baylor 1997; Kuffler 1953; Nirenberg and Meister 1997; Stone and Pinto 1993). We found that the cells did divide into classes with these parameters, although there were some departures from the standard models. First, only a subset of cells, those with short latency responses, showed division into ON, OFF, and ON-OFF classes. Second, only the short latency on cells showed division into transient and sustained types. Assessing subdivision by duration for the OFF and ON-OFF cells was not possible, because these groups contained too few cells for analysis.

Next, we tested whether ganglion cells clustered by degree of nonlinearity in the stimulus-to-response transformation, and found that the result did not conform to previous predictions (Stone and Pinto 1993) - the cells did not cluster into linear (X-like) and nonlinear (Y-like) groups. Instead, they formed a continuum with varying degrees of nonlinearity in their responses.

This was a surprising result. One possibility is that it occurred because data across all eccentricities were included, following Hochstein and Shapley (1976). In studies of dendritic tree size, a property associated with degree of nonlinearity, clusters occur only when eccentricity is taken into account - at least in cat, where it has been examined in detail (Boycott and Wassle 1974). Thus we tested whether the linear/ nonlinear distinction might appear if we focused our analysis on a narrower region of retina and used the region immediately adjacent to the optic nerve. [In mouse, the optic nerve is very close - within a few hundred microns - of central retina (Jeon et al. 1998; Sun et al. 2002).] We found that it $\operatorname{did} \operatorname{not}(P=$ $0.07, n=36$ ) (Fig. $8 B$ ). Note that the only dips in the distribution were well above 1 , the index value that would be expected to separate linear and nonlinear cells.

We also tested whether the linear/nonlinear distinction 
would appear if we used alternate versions of the nonlinearity index that have been proposed (Mechler and Ringach 2002; So and Shapley 1979), such as the log of the index, the F2/F1 ratio, and the log of the F2/F1 ratio (where $\mathrm{F} 1$ and $\mathrm{F} 2$ are the amplitudes of the first and second harmonic, and the maximum ratio is used as the F2/F1 ratio). These also did not reveal a division. Analyses using these versions all gave the same answer: ganglion cells do not divide into distinct linear and nonlinear groups in the mouse retina, whether evaluated as a whole population or as a subpopulation near the optic nerve. (Using data from the whole population: for the log of the nonlinearity index, unimodality could not be rejected, $P=$ 0.39 ; for the F2/F1 ratio, $P=0.48$; for the $\log$ of the $\mathrm{F} 2 / \mathrm{F} 1$ ratio, $P=0.44$. Using data from the optic nerve region: for the $\log$ of the nonlinearity index, unimodality could not be rejected, $P=0.26$; for the $\mathrm{F} 2 / \mathrm{F} 1$ ratio, $P=0.11$; for the $\log$ of the F2/F1 ratio, $P=0.22$.)

\section{Number of cell classes detected constitutes a lower bound}

The most rigorous way to identify cell classes is to characterize them with respect to several parameters and then evaluate then simultaneously-that is, construct an $n$-dimensional plot, where $n$ is the number of parameters, and then test for clustering in $n$-dimensional space (Rodieck and Brening 1983). Unfortunately, this approach is not currently possible because a prohibitively large amount of data is required-the amount of data needed scales exponentially with the number of parameters. For this reason, we used a serial approach. We started with one parameter and tested the distribution of that parameter for division into clusters; we then chose another, and so forth. Whenever clusters appeared in a distribution, we tested each separately for further clustering along all other parameters. This approach was repeated in several different orders to explore as much of parameter space as possible (for further detail, see METHODS).

Although this approach covers a lot of ground, it is not complete-clusters can be missed. For example, some clusters may be detectable using only 2 parameters at once, that is, by plotting the data in two dimensions (e.g., see Boycott and Wassle 1974). If such clusters exist, they would be missed by the serial analysis. For this reason, the number of cell classes we identified should be regarded as a lower bound on the total number that exists with a given stimulus and set of response parameters. Thus with the spot stimulus and the parameters of latency, duration and relative amplitude of the ON and OFF responses, 5 clusters were identified, but the possibility remains that multidimensional analysis would have revealed more. Along the same lines, with the grating stimulus and the nonlinearity index, no divisions were found, but the possibility remains that the index would produce clusters if evaluated simultaneously with other parameters. These possibilities may soon be testable as techniques for mass screening of neuronal activity (e.g., genetic sensors, activity-dependent dyes) become more advanced and expand the size of data sets (Nakai et al. 2001; Smetters et al. 1999; Yu et al. 2003; Zochowski 2000).

\section{Importance of cell classification}

Why is it important to know, using an objective approach, how ganglion cell responses sort? There are 3 main reasons.
First, this information is needed for building reliable quantitative models of downstream processing. The output responses of the retina serve as the building blocks for circuits in higher brain areas, and different building blocks lead to different model predictions. Our findings provide evidence that models of downstream processing that assume separate ON and OFF inputs (e.g., for constructing on and ofF subregions of cortical receptive fields) are applicable to the mouse visual system (Hubel and Wiesel 1962; Palmer and Davis 1981; Schiller, 1982; Worgotter and Koch 1991), although some adjustments may be needed. Specifically, the on and off classes are made up of broader distributions than are typically assumed, which will have bearing on model predictions. In contrast, models invoking separate linear and nonlinear channels at the level of the retina (Mastronarde 1987a,b; Shapley and Perry 1986; Sherman 1985) may not be readily applicable to the mouse, given that we were unable to find clear evidence of distinct linear and nonlinear classes. This is consistent with findings in primate, where evidence for distinct linear and nonlinear classes is also weak or not present (Derrington and Lennie 1984; Usrey and Reid 2000). When distributions of nonlinearity indices were evaluated in the magnocellular and parvocellular pathways, no visible gaps emerged (Derrington and Lennie 1984). Likewise, when the data from the two pathways are superimposed, no visible gaps appear. These results suggest that models based on the linear/nonlinear scheme may be less applicable across species than previously thought, whereas those based on the on versus OFF scheme may be universal.

The second reason it is important to have objective information about how ganglion cell responses in the mouse retina sort is that it guides us as to how circuits in this species are laid out. For example, the fact that there are distinct transient and sustained groups for the on channel indicates that there are two separate on microcircuits. Likewise, the fact that there are two ON latency groups also implies that there are two separate microcircuits. In addition, the finding that there are 2 classes of cells that show responses to the light and dark spots, the short latency ON-OFF cells and the long latency cells, indicates that there are 2 places in the circuitry where ON and OFF pathways converge (see Masland et al. 1984).

Finally, the third reason, it is important to have a quantitative description of visual responses and their classification is that it provides a baseline for comparison with mutant or perturbed retinas, such as models of retinitis pigmentosa (An et al. 2002; Strettoi et al. 2002), night blindness (Gregg et al. 2003; Pardue et al. 1998), microphthalmia (Green et al. 2003; Steingrimsson et al. 1994), and knockout mice that lack specific receptors or neurons (Masu et al. 1995; McCall et al. 1996, 2002; Nirenberg and Meister 1997; Soucy et al. 1998) or show developmental defects in their circuitry (Bansal et al. 2000; Hammang et al. 1993; Wang et al. 2002). This baseline is also critical for measuring the success of treatments for such defects, such as retinal transplantation (Litchfield et al. 1997; Radner et al. 2001, 2002), regeneration (Porciatti et al. 1996; Travis et al. 1992), or prostheses (Humayun 2001; Margalit et al. 2002), which aim to duplicate normal visual responses. By defining cell classes as clusters in an easy-to-measure parameter space, we provide an objective description of what normal visual responses are, which can be retraced exactly by other investigators. 


\section{Further classifications}

Many other properties, besides those investigated here, both physiological and anatomical, have been used to classify ganglion cells in other species and are likely to be important for classifying ganglion cells in mouse as well, such as direction selectivity, orientation selectivity (Amthor 1989a,b; Cleland and Levick 1974), intrinsic membrane properties (membrane time constant, input resistance, resting potential) (O'Brien et al. 2002), aspects of the autocorrelation function (DeVries and Baylor 1997), degree of rod input (Deans et al. 2002; Sterling 1983), sensitivity to 2-amino-4-phosphonobutyric acid (Slaughter and Miller 1981), and numerous anatomical properties, such as depth of ramification in the inner plexiform layer (Dacey et al. 2003; Famiglietti and Kolb 1976; Nelson et al. 1978; Pang et al. 2002; Rockhill et al. 2002; Roska and Werblin, 2001), soma size, dendritic diameter, branching density, dendritic coverage (Amthor et al. 1989a,b; Boycott and Wassle 1974; Dacey et al. 2003; Rockhill et al. 2002; Sun et al. 2002), synaptic input (Calkins et al. 1998), and location of projection in target tissue (Vaney et al. 1981). Cluster analysis using these parameters will provide a way to test their roles in mouse classification.

Recent studies specifically in mouse suggest that more physiological classes are likely to be found. Using morphological properties (depth of stratification, soma size and shape, and dendritic field size and branching patterns), Sun et al. (2002) posited 14 types, suggesting that more stimuli and response parameters will be needed to complete the physiological characterization. In addition, Deans et al. (2002) suggest that degree of rod input provides a basis for division of on cells; thus there may be subdivisions, using this parameter, among the clusters we found.

We thank R. Shapley, P. Latham, and D. Ringach for helpful discussion and comments on the manuscript.

\section{I S C L O S URES}

This work was supported by grants from the Beckman Foundation, the Klingenstein Fund, the Whitehall Foundation, and by a grant from National Institutes of Health to S. Nirenberg.

\section{REFERENCES}

Abbott LF and Chance FS. Rethinking the taxonomy of visual neurons. Nat Neurosci 5: 391-392, 2002.

Amthor FR, Takahashi ES, and Oyster CW. Morphologies of rabbit retinal ganglion cells with concentric receptive fields. J Comp Neurol 280: 72-96, 1989a.

Amthor FR, Takahashi ES, and Oyster CW. Morphologies of rabbit retinal ganglion cells with complex receptive fields. J Comp Neurol 280: 97-121, 1989 b.

An GJ, Asayama N, Humayun MS, Weiland J, Cao J, Kim SY, Grebe R, de Juan E Jr, and Sadda S. Ganglion cell responses to retinal light stimulation in the absence of photoreceptor outer segments from retinal degenerate rodents. Curr Eye Res 24: 26-32, 2002.

Balkema GW and Pinto LH. Electrophysiology of retinal ganglion cells in the mouse: a study of a normally pigmented mouse and a congenic hypopigmentation mutant, pearl. J Neurophys 48: 968-982, 1982.

Bansal A, Singer JH, Hwang BJ, Xu W, Beaudet A, and Feller MB. Mice lacking specific nicotinic acetylcholine receptor subunits exhibit dramatically altered spontaneous activity patterns and reveal a limited role for retinal waves in forming ON and OFF circuits in the inner retina. $J$ Neurosci 20: 7672-7681, 2000.

Barlow HB. Summation and inhibition in the frog's retina. J Physiol 119: 69-88, 1953.
Boycott BB and Wassle H. The morphological types of ganglion cells of the domestic cat's retina. J Physiol 240: 397-419, 1974.

Calkins DJ, Tsukamoto Y, and Sterling P. Microcircuitry and mosaic of a blue-yellow ganglion cell in the primate retina. J Neurosci 18: 3375-3385, 1998.

Cleland BG, Dubin MW, and Levick WR. Sustained and transient neurones in the cat's retina and lateral geniculate nucleus. J Physiol 217: 473-496, 1971.

Cleland BG and Levick WR. Brisk and sluggish concentrically organized ganglion cells in the cat's retina. J Physiol 240: 421-456, 1974.

Dacey DM, Peterson BB, Robinson FR, and Gamlin PD. Fireworks in the primate retina: in vitro photodynamics reveals diverse LGN-projecting ganglion cell types. Neuron 37: 15-27, 2003.

Deans MR, Volgyi B, Goodenough DA, Bloomfield SA, and Paul DL. Connexin36 is essential for transmission of rod-mediated visual signals in the mammalian retina. Neuron 36: 703-712, 2002.

Derrington AM and Lennie P. Spatial and temporal contrast sensitivities of neurones in lateral geniculate nucleus of macaque. J Physiol 357: 219-240, 1984.

DeVries SH and Baylor DA. Mosaic arrangement of ganglion cell receptive fields in rabbit retina. J Neurophysiol 78: 2048-2060, 1997.

Dodd RL. The Role of Arrestin and Recoverin in Signal Transduction by Retinal Rod Photoreceptors (PhD dissertation). Palo Alto, CA: Stanford University, 1998.

Efron B and Tibshirani RJ. An Introduction to the Bootstrap. New York: Chapman \& Hall, 1993.

Enroth-Cugell C and Robson JG. The contrast sensitivity of retinal ganglion cells of the cat. $J$ Physiol 187: 517-552, 1966.

Famiglietti EV and Kolb H. Structural basis for ON- and OFF-center responses in retinal ganglion cells. Science 194: 193-195, 1976.

Green ES, Stubbs JL, and Levine EM. Genetic rescue of cell number in a mouse model of microphthalmia: interactions between Chx10 and G1-phase cell cycle regulators. Development 130: 539-552, 2003.

Gregg RG, Mukhopadhyay S, Candille SI, Ball SL, Pardue MT, McCall MA, and Peachey NS. Identification of the gene and the mutation responsible for the mouse nob phenotype. Invest Ophthalmol Vis Sci 44: 378-384, 2003.

Hammang JP, Behringer RR, Baetge EE, Palmiter RD, Brinster RL, and Messing A. Oncogene expression in retinal horizontal cells of transgenic mice results in a cascade of neurodegeneration. Neuron 10: 1197-209, 1993.

Hartline HK. The response of single optic nerve fibers of the vertebrate eye to illumination of the retina. Am J Physiol 121: 400-415, 1938.

Hochstein S and Shapley RM. Quantitative analysis of retinal ganglion cell classifications. J Physiol 262: 237-264, 1976.

Hubel DH and Wiesel TN. Receptive fields, binocular interaction and functional architecture in the cat's visual cortex. J Physiol 160: 106-154, 1962.

Humayun MS. Intraocular retinal prosthesis. Trans Am Ophthalmol Soc 99: 271-300, 2001.

Jeon CJ, Strettoi E, and Masland RH. The major cell populations of the mouse retina. J Neurosci 18: 8936-8946, 1998.

Kuffler SW. Discharge patterns and functional organization of mammalian retina. J Neurophysiol 16: 37-68, 1953.

Litchfield TM, Whiteley SJO, and Lund RD. Transplantation of retinal pigment epithelial, photoreceptor and other cells as a treatment for retinal degeneration. Exp Eye Res 64: 655-666, 1997.

Marc RE and Jones BW. Molecular phenotyping of retinal ganglion cells. J Neurosci 22: 413-427, 2002.

Margalit E, Maia M, Weiland JD, Greenberg RJ, Fujii GY, Torres G, Piyathaisere DV, O'Hearn TM, Liu W, Lazzi G, Dagnelie G, Scribner DA, de Juan E Jr, and Humayun MS. Retinal prosthesis for the blind. Surv Ophthalmol 47: 335-356, 2002.

Masland RH, Mills JW, and Cassidy $\mathbf{C}$. The functions of acetylcholine in the rabbit retina. Proc R Soc Lond B Biol Sci 223: 121-139, 1984.

Mastronarde DN. Two classes of single-input X-cells in cat lateral geniculate nucleus. I. Receptive-field properties and classification of cells. J Neurophysiol 57: 357-380, 1987a.

Mastronarde DN. Two classes of single-input X-cells in cat lateral geniculate nucleus. II. Retinal inputs and the generation of receptive field properties. J Neurophysiol 57: 381-413, 1987b.

Masu M, Iwakabe H, Tagawa Y, Miyoshi T, Yamashita M, Fukuda Y, Sasaki H, Hiroi K, Nakamura Y, Shigemoto R, Takada M, Nakamura K, Nakao K, Katsuki M, and Nakanishi S. Specific deficit of the ON response in visual transmission by targeted disruption of the mGluR6 gene. Cell 80: 757-765, 1995 
McCall MA, Gregg RG, Merriman K, Goto Y, Peachey NS, and Stanford LR. Morphological and physiological consequences of the selective elimination of rod photoreceptors in transgenic mice. Exp Eye Res 63: 35-50, 1996.

McCall MA, Lukasiewicz PD, Gregg RG, and Peachey NS. Elimination of the $\rho 1$ subunit abolishes GABAc receptor expression and alters visual processing in the mouse retina. $J$ Neurosci 22: 4163-4174, 2002

Mechler F and Ringach DL. On the classification of simple and complex cells. Vision Res 42: 1017-1033, 2002.

Meister M, Pine J, and Baylor DA. Multi-neuronal signals from the retina: acquisition and analysis. J Neurosci Methods 51: 95-106, 1994.

Nakai J, Ohkura M, and Imoto KA. A high signal-to-noise $\mathrm{Ca}(2+)$ probe composed of a single green fluorescent protein. Nat Biotech 19: 37-141, 2001.

Nelson R, Famiglietti EV, and Kolb H. Intracellular staining reveals different levels of stratification for ON- and OFF-center ganglion cells in cat retina. J Neurophysiol 41: 472-483, 1978.

Nirenberg S, Carcieri SM, Jacobs AL, and Latham PE. Retinal ganglion cells act largely as independent encoders. Nature 411: 698-701, 2001.

Nirenberg $\mathbf{S}$ and Meister $\mathbf{M}$. The light response of retinal ganglion cells is truncated by a displaced amacrine circuit. Neuron 18: 637-650, 1997.

O'Brien BJ, Isayama T, Richardson R, and Berson DM. Intrinsic physiological properties of cat retinal ganglion cells. J Physiol 538: 787-802, 2002.

Palmer LA and Davis TL. Receptive-field structure in cat striate cortex. J Neurophysiol 46: 260-267, 1981.

Pang JJ, Gao F, and Wu SM. Segregation and integration of visual channels: layer-by-layer computation of ON-OFF signals by amacrine cell dendrites. J Neurosci 22: 4693-4701, 2002.

Pardue MT, McCall MA, LaVail MM, Gregg RG, and Peachey NS. A naturally occurring mouse model of X-linked congenital stationary night blindness. Invest Ophthalmol Vis Sci 39: 2443-2449, 1998.

Peichl $\mathbf{L}$ and Wassle H. Morphological identification of ON- and OFF-centre brisk transient (Y) cells in the cat retina. Proc R Soc Lond B Biol Sci 212: 139-156, 1981.

Porciatti V, Pizzorusso T, Cenni MC, and Maffei L. The visual response of retinal ganglion cells is not altered by optic nerve transection in transgenic mice overexpressing Bcl-2. Proc Natl Acad Sci USA 93: 14955-14959, 1996.

Radner W, Sadda SR, Humayun MS, Suzuki S, and de Juan E Jr. Increased spontaneous retinal ganglion cell activity in rd mice after neural retinal transplantation. Invest Ophthalmol Vis Sci 43: 3053-3058, 2002.

Radner W, Sadda SR, Humayun MS, Suzuki S, Melia M, Weiland J, and de Juan E Jr. Light-driven retinal ganglion cell responses in blind rd mice after neural retinal transplantation. Invest Ophthalmol Vis Sci 42: 1057$1065,2001$.

Rockhill RL, Daly FJ, MacNeil MA, Brown SP, and Masland RH. The diversity of ganglion cells in the mammalian retina. $J$ Neurosci 22: 38313843, 2002.

Rodieck RW. The First Steps in Seeing. Sunderland, MA: Sinauer, 1998.

Rodieck RW and Brening RK. Retinal ganglion cells: properties, types, genera, pathways and trans-species comparisons. Brain Behav Evol 23: 121-164, 1983.

Roska B and Werblin F. Vertical interactions across ten parallel, stacked representations in the mammalian retina. Nature 410: 583-587, 2001.

Rowe MH and Stone J. Naming of neurons: classification and naming of cat retinal ganglion cells. Brain Behav Evol 14: 185-216, 1977.

Schiller PH. Central connections of the retinal on and OFF pathways. Nature. 297: 582-583, 1982.

Shapley R and Perry VH. Cat and monkey retinal ganglion cells and their visual functional roles. Trends Neurosci 9: 229-235, 1986.
Sherman SM. Functional organization of the W-, X-, and Y-cell pathways in the cat: a review and hypothesis. In: Progress in Psychobiology and Physiological Psychology. New York: Academic, 1985, p. 233-314.

Silverman BW. Using kernel density estimates to investigate multimodality. $J$ $R$ Stat Soc B: 97-99, 1981.

Silverman BW. Density estimation for statistics and data analysis. In: Monographs on Statistics and Applied Probability. London: Chapman \& Hall, 1986.

Slaughter MM and Miller RF. 2-Amino-4-phosphonobutyric acid: a new pharmacological tool for retina research. Science 211: 183, 1981.

Smetters D, Majewska A, and Yuste R. Detecting action potentials in neuronal populations with calcium imaging. Methods: A Companion to Methods Enzymol 2: 215-221, 1999.

So YT and Shapley R. Spatial properties of X and Y cells in the lateral geniculate nucleus of the cat and conduction velocities of their inputs. Exp Brain Res 36: 533-550, 1979.

Soucy E, Wang Y, Nirenberg S, Nathans J, and Meister M. A novel signaling pathway from rod photoreceptors to ganglion cells in mammalian retina. Neuron 21: 481-493, 1998.

Stanford LR. X-cells in the cat retina: relationships between the morphology and physiology of a class of cat retinal ganglion cells. J Neurophysiol 58 : 940-964, 1987.

Steingrimsson E, Moore KJ, Lamoreux ML, Ferre-D'Amare AR, Burley SK, Zimring DC, Skow LC, Hodgkinson CA, Arnheiter H, Copeland NG, and Jenkins NA. Molecular basis of mouse microphthalmia (mi) mutations helps explain their developmental and phenotypic consequences. Nat Genet 8: 256-263, 1994.

Sterling P. Microcircuitry of the cat retina. Апnи Rev Neurosci 6: 149-185, 1983.

Stone C and Pinto LH. Response properties of ganglion cells in the isolated mouse retina. Vis Neurosci 10: 31-39, 1993.

Stone J. Parallel Processing in the Visual System. New York: Plenum, 1983.

Strettoi E, Porciatti V, Falsini B, Pignatelli V, and Rossi C. Morphological and functional abnormalities in the inner retina of the $\mathrm{rd} / \mathrm{rd}$ mouse. $\mathrm{J} \mathrm{Neu}$ rosci 22: 5492-5504, 2002.

Sun W, Li N, and He S. Large-scale morphological survey of mouse retinal ganglion cells. J Comp Neurol 451: 115-126, 2002.

Travis GH, Groshan KR, Lloyd M, and Bok D. Complete rescue of photoreceptor dysplasia and degeneration in transgenic retinal degeneration slow (rds) mice. Neuron 9: 113-119, 1992.

Usrey WM and Reid RC. Visual physiology of the lateral geniculate nucleus in two species of New World monkey: Saimiri sciureus and Aotus trivirgatis. J Physiol 523: 755-769, 2000.

Vaney DI, Peichl L, Wassle H, and Illing RB. Almost all ganglion cells in the rabbit retina project to the superior colliculus. Brain Res 212: 447-453, 1981

Wang YP, Dakubo G, Howley P, Campsall KD, Mazarolle CJ, Shiga SA, Lewis PM, McMahon AP, and Wallace VA. Development of normal retinal organization depends on Sonic hedgehog signaling from ganglion cells. Nat Neurosci 5: 831-832, 2002.

Wassle $\mathbf{H}$ and Boycott BB. Functional architecture of the mammalian retina. Physiol Rev 71: 447-480, 1991.

Worgotter F and Koch C. A detailed model of the primary visual pathway in the cat: comparison of afferent excitatory and intracortical inhibitory connection schemes for orientation selectivity. J Neurosci 11: 1959-1979, 1991.

Yu D, Baird GS, Tsien RY, and Davis RJ. Detection of calcium transients in Drosophila mushroom body neurons with camgaroo reporters. $J$ Neurosci 23: 64-72, 2003.

Zochowski M, Wachowiak M, Falk CX, Cohen LB, Lam YW, Antic S, and Zecevic D. Imaging membrane potential with voltage-sensitive dyes. Biol Bull 198: 1-21, 2000. 